\title{
Frames and Topological Algebras for a Double-Power Monad
}

\author{
GIULIA FROSONI \\ GIUSEPPE ROSOLINI \\ ALESSIO SANTAMARIA
}

\begin{abstract}
We study the algebras for the double power monad on the Sierpiński space in the Cartesian closed category of equilogical spaces and produce a connection of the algebras with frames. The results hint at a possible synthetic, constructive approach to frames via algebras, in line with that considered in Abstract Stone Duality by Paul Taylor and others.
\end{abstract}

2010 Mathematics Subject Classification 18D15 (primary); 06D22 54B30 (secondary)

Keywords: Equilogical space, frame, double-power monad

\section{Introduction}

The category $\mathcal{E} q u$ of equilogical spaces introduced by Dana Scott in [24] offers a very nice extension of the category $\mathcal{T}_{o p_{0}}$ of $\mathrm{T}_{0}$-spaces and continuous maps, as it is a locally Cartesian closed quasitopos and the embedding of $\mathrm{T}_{0}$-spaces is full and preserves all products and existing exponentials. In other words, one may work with $\mathrm{T}_{0}$-spaces as if they formed a Cartesian closed category, just that sometimes the necessary space need not be topological, but it is just equilogical; see also Bauer, Birkedal and Scott [1].

For instance, the Sierpiński space $S$ is the open-subset classifier, in the sense that given a $\mathrm{T}_{0}$-space $T$, for every $\mathrm{T}_{0}$-space $X$ a continuous map $f: X \longrightarrow \mathrm{S}^{T}$ determines precisely an open subset of the space $X \times T$. But there is a problem in reading the previous sentence: the object $S^{T}$ need not exist as a topological space. The immediate solution is to read that sentence in the category of equilogical spaces where $S^{T}$ always exists; it is just that it may be a true equilogical space (ie which is not topological).

Such an extension of the language of Cartesian closed categories (and of the $\lambda$-calculus) was tested in various guises in many papers, see for instance Taylor [28, 32] and Vickers and Townsend [34]. In particular, in Bucalo and Rosolini [5] that extension is used to prove an intrinsic description of the soberification of a $\mathrm{T}_{0}$-space which involved 
the monad on the double power of $\mathrm{S}$ - the action on an object $E$ is $\mathrm{S}^{\left(\mathrm{S}^{E}\right)}$ - which has also been studied as an instance of a continuation monad; see Thielecke [33]. All this directed to a study of the category of algebras for the monad on the double power of $\mathrm{S}$ which is what we confront with in the present paper. ${ }^{1}$

Bucalo and Rosolini [5] suggested that the algebras for the monad $\mathbf{S}^{\mathbf{2}}$ resembled frames with frame homomorphisms and we address exactly that connection in the present paper. We show that the structure of $\mathbf{S}^{\mathbf{2}}$-algebra on an equilogical space $A$ gives rise to a structure of frame on the set of global sections of $A$. In order to do that, we also study some $\mathcal{E} q u$-enriched Lawvere theories, in the sense of Power [21], which relate to $\mathbf{S}^{\mathbf{2}}$-algebras and provides a description of frames internal to the category $\mathcal{E} q u$, and we characterise the enriched cotensor-preserving functors from the enriched algebraic theory $\mathcal{L}(\mathbf{M})$ of an enriched monad $\mathbf{M}$ in a way that extends results of Dubuc and Power, see Dubuc [7] and [21]. This makes it possible, in particular, to fit the example of soberification of $\mathrm{T}_{0}$-spaces precisely within the paradigm considered in Paul Taylor's Abstract Stone Duality; see Taylor [27, 28, 29, 32] and Vickers and Townsend [34].

In Section 2 we recall the category $\mathcal{E} q u$ of equilogical spaces as a Cartesian closed extension of the category $\mathcal{T}_{o} p_{0}$ of $\mathrm{T}_{0}$-spaces and continuous maps, and in Section 3 we present the double power monad $\mathbf{S}^{\mathbf{2}}$ on equilogical spaces determined by the Sierpiński space S. In Section 4 we introduce the enriched algebraic theory related to an enriched monad on a Cartesian closed category and to the monad $\mathbf{S}^{\mathbf{2}}$ in $\mathcal{E} q u$ in particular. We prove a characterisation of the enriched cotensor-preserving functors from the enriched algebraic theory $\mathcal{L}(\mathbf{M})$ of an enriched monad $\mathbf{M}$ in Theorem 4.11; we also give a different presentation of the theory $\mathcal{L}\left(\mathbf{S}^{2}\right)$ which will be applied in Section 5, where we prove some internal properties of $\mathbf{S}^{\mathbf{2}}$-algebras in $\mathcal{E} q u$. In particular we show that every $\mathbf{S}^{\mathbf{2}}$-algebra is an internal frame in $\mathcal{E} q u$ in Corollary 5.7 and show how their global sections are connected to frames in Corollary 5.8.

\section{Basic properties of equilogical spaces}

We adopt the notation in Frosoni and Rosolini [10] and we refer the reader there for a survey of the basic results. The reader is also referred to Bauer, Birkedal, and Scott [1], Birkedal, Carboni, Rosolini and Scott [2] and Scott [24].

Recall that an equilogical space $E=\left(|E|, \tau_{E}, \equiv_{E}\right)$ consists of a $\mathrm{T}_{0}$-space $\left(|E|, \tau_{E}\right)$ and an equivalence relation $\equiv_{E} \subseteq|E| \times|E|$ on the points of the space.

\footnotetext{
${ }^{1}$ We shall follow Taylor [32] and write $\mathbf{S}^{\mathbf{2}}$ for that monad; see Section 3.
} 
A map of equilogical spaces $[f]: E \longrightarrow F$ is an equivalence class of continuous functions

$$
f:\left(|E|, \tau_{E}\right) \longrightarrow\left(|F|, \tau_{F}\right)
$$

preserving the equivalence relations, ie if $x \equiv_{E} x^{\prime}$ then $f(x) \equiv_{F} f\left(x^{\prime}\right)$ for all $x$ and $x^{\prime}$ in $|E|$, where two such continuous functions $f, f^{\prime}:\left(|E|, \tau_{E}\right) \longrightarrow\left(|F|, \tau_{F}\right)$ are equivalent if $f(x) \equiv_{F} f^{\prime}(x)$ for all $x \in|E|$.

Composition of maps of equilogical spaces $[f]: E \longrightarrow F$ and $[g]: F \longrightarrow G$ is given on (any of) their continuous representatives: $[g] \circ[f]:=[g \circ f]$.

The data above determine the category $\mathcal{E} q u$ of equilogical spaces which is an extension of the category of $\mathrm{T}_{0}$-spaces: the embedding

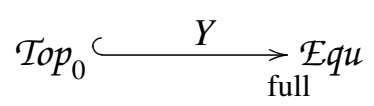

maps a $\mathrm{T}_{0}$-space $(T, \tau)$ to the equilogical space on $(T, \tau)$ with the diagonal relation, ie the equilogical space $(T, \tau,=)$.

As shown in [24] the category $\mathcal{E} q u$ is equivalent to the category $\mathcal{P E} \mathcal{E} u$, where an object is a pair $P=\left(L_{P}, \approx_{P}\right)$ consisting of an algebraic lattice $L_{P}$ and a symmetric and transitive relation $\approx_{P} \subseteq\left|L_{P}\right| \times\left|L_{P}\right|$ on $\left|L_{P}\right|$, ie a partial equivalence relation on $\left|L_{P}\right|$. An arrow in $\mathcal{P E} q u[g]: P \longrightarrow Q$ is an equivalence class of Scott-continuous functions $g: L_{P} \longrightarrow L_{Q}$ such that whenever $a \approx_{P} b$, also $g(a) \approx_{Q} g(b)$, where two such continuous functions $g, g^{\prime}: L_{P} \longrightarrow L_{Q}$ are equivalent if $g(a) \approx_{Q} g^{\prime}(a)$ for all $a \approx_{P} a$.

The composition of two arrows $[g]: P \longrightarrow Q$ and $[h]: Q \longrightarrow R$ in $\mathcal{P E} q u$ is given on (any of) their continuous representatives: $[h] \circ[g]:=[h \circ g]$.

To describe the equivalence of categories, for an object $P$ in $\mathcal{P E} q u$ write $\mathrm{D}_{P}$ for the domain $\left\{x \in\left|L_{P}\right| \mid x \approx_{P} x\right\}$ of the relation $\approx_{P}$ and note that $\approx_{P} \subseteq \mathrm{D}_{P} \times \mathrm{D}_{P}$. Also write $\tau_{\mathrm{Sc}}$ for the Scott topology on the algebraic lattice $L_{P}$ and $\tau_{\text {sub }}$ for the subspace topology induced by the inclusion $\mathrm{D}_{P} \subseteq\left|L_{P}\right|$. We can now recall the two results from [24] crucial for the developments in the paper.

Proposition 2.1 The assignment

$$
\begin{aligned}
& \mathcal{P E} q u \stackrel{Z}{\longrightarrow} \underset{\mathcal{E} q u}{\longrightarrow}\left(\mathrm{D}_{P}, \tau_{\mathrm{sub}}, \approx_{P}\right) \\
& P \longmapsto
\end{aligned}
$$

extends to a functor which is an equivalence of categories. 
Proposition 2.2 Let $P=\left(L_{P}, \approx_{P}\right)$ and $Q=\left(L_{Q}, \approx_{Q}\right)$ be objects in $\mathcal{P} \mathcal{E} q u$. Then

(i) their product can be chosen as

$$
P \times Q=\left(L_{P} \times L_{Q}, \approx_{P \times Q}\right)
$$

where $\langle a, b\rangle \approx_{P \times Q}\left\langle a^{\prime}, b^{\prime}\right\rangle$ if $a \approx_{P} a^{\prime}$ and $b \approx_{Q} b^{\prime}$, and

(ii) their exponential can be chosen as $Q^{P}=\left(\operatorname{Cont}\left(L_{P}, L_{Q}\right), \approx_{Q^{P}}\right)$ where $f \approx_{Q^{P}} f^{\prime}$ if, for every $a, a^{\prime} \in\left|L_{P}\right|$, whenever $a \approx_{P} a^{\prime}$ it is $f(a) \approx_{Q} f^{\prime}\left(a^{\prime}\right)$.

Note that via the equivalence in Proposition 2.1 an object $P$ of $\mathcal{P E} q u$ gives rise to a diagram:

$$
Z(P) \stackrel{\left[\operatorname{id}_{\mathrm{D}_{P}}\right]}{\longrightarrow}\left(\mathrm{D}_{P}, \tau_{\mathrm{sub}},=\right) \succ\left(\left|L_{P}\right|, \tau_{\mathrm{Sc}},=\right)
$$

Remark 2.3 The category $\mathcal{P E} q u$ is (equivalent to) the quotient completion of the subset doctrine on the category $\mathcal{A} \mathcal{L}$ of algebraic lattices and Scott-continuous functions; see Maietti and Rosolini [18, 19, 20] and Rosolini and Streicher [22].

\section{The monad of the double power}

Consider the Sierpiński $\mathrm{T}_{0}$-space $\mathrm{S}$ which consists of two points $\{\top, \perp\}$ and one non-trivial open subset $\{T\}$. In other words, one point is open, the other is closed. It is an algebraic lattice with the Scott topology. So the equilogical space $Y(\mathrm{~S})=\left(|\mathrm{S}|, \tau_{\mathbf{S}},=\right)$ is a regular projective of $\mathcal{E} q u$.

We shall concentrate on the Sierpiński space as an object of $\mathcal{E} q u$ as we intend to study the algebraic theory of S, and for that we need a Cartesian closed category. That theory played a crucial role in a synthetic presentation of the soberification of a topological space in Bucalo and Rosolini [5, 4] as it showed that the notion of soberification is intrinsically related to the topology and to a monad derived from S. From now on we shall write the equilogical space $Y(\mathrm{~S})$ simply as $\mathrm{S}$, dropping the $Y$.

The main object of our study fits very well within a paradigm which was studied in depth in general category-theoretical terms, in particular we refer the reader to a series of papers by Paul Taylor [27, 28, 29, 30, 31, 32], to Bucalo and Rosolini [3] and to Dubuc [7]. We develop the basic details in an ambient category which is Cartesian closed. Since typed $\lambda$-calculus is the internal language of Cartesian closed categories (see Lambek and Scott [15]) we shall use it extensively in the following. 
Let $C$ be a Cartesian closed category, eg the category $\mathcal{E} q u$, and let $O$ be a fixed object in $\mathcal{C}$, eg $\mathrm{S}$ in $\mathcal{E} q u$.

Since the functor $O^{(-)}: C \rightarrow C^{\text {op }}$ is self-adjoint-explicitly

$$
C \underset{O^{(-)}}{\stackrel{O^{(-)}}{\stackrel{\perp}{\longleftarrow}}} C^{\mathrm{op}}
$$

-it gives rise to a monad on $C$. The functor part of the monad sends an arbitrary object $C$ of $C$ to the object $O^{\left(O^{C}\right)}$. The unit of the monad has components $\eta_{C}: C \longrightarrow O^{\left(O^{C}\right)}$, the exponential adjunct of the composite

$$
C \times O^{C} \stackrel{\left\langle\mathrm{pr}_{2}, \mathrm{pr}_{1}\right\rangle}{\longrightarrow} O^{C} \times C \stackrel{\mathrm{ev}}{\longrightarrow} O
$$

which, in $\lambda$-notation using - to denote application, is written:

$$
\lambda F: O^{C} . F \cdot x \text { in context } x: C
$$

The multiplication component $\mu_{C}: O^{\left(O^{\left(O^{(O)}\right)}\right)} \longrightarrow O^{\left(O^{C}\right)}$ is the map $O^{\eta_{O^{C}}}$, which is:

$$
\lambda F: O^{C} \cdot G \cdot\left(\lambda U: O^{\left(O^{C}\right)} \cdot U \cdot F\right) \quad \text { in context } G: O^{\left(O^{\left(O^{\left(O^{C}\right)}\right)}\right)}
$$

The climbing exponentials are unpleasant to read and we follow Taylor lowering the exponent of the functor-so we write $O(C)$ in place of $O^{C}$ - and denoting iterations as $O^{2}(C), O^{3}(C) \ldots$ which replace $O^{\left(O^{C}\right)}, O^{\left(O^{\left(O^{C}\right)}\right)} \ldots$ In particular, we write the monad as $\mathbf{O}^{2}$.

Examples 3.1 A well-known example of this kind of monads is obtained when one takes the category Set of sets and functions as $\mathcal{C}$ and the set $D=\{0,1\}$ as the object $O$. The Eilenberg-Moore category of algebras for the monad $\mathbf{D}^{\mathbf{2}}$ is that of complete Boolean algebras.

Another example is with $\mathcal{C}$ the category $\mathcal{P}$ os of posets and monotone functions, the object $O$ is the standard order $P$ on the previous set $D$. The Eilenberg-Moore category of algebras for the monad $\mathbf{P}^{\mathbf{2}}$ is that of completely distributive lattices.

An example is also that where $O$ is again the poset $P$, but in a different category from the previous one: $C$ is the category $\mathcal{D P}$ os of posets with sups of directed subsets and functions preserving sups of directed subsets. The Eilenberg-Moore algebras for the monad $\mathbf{P}^{\mathbf{2}}$ on the category $\mathcal{D} \mathcal{P}$ ss is that of frames and frame homomorphisms. 
In order to present some properties of the monad $\mathbf{S}^{\mathbf{2}}$ on $\mathcal{E} q u$, it is useful to introduce auxiliary full subcategories of $\mathcal{P E} q u$. We denote $\mathcal{R} \mathcal{P} \mathcal{E} q u$ the full subcategory of $\mathcal{P E} q u$ on those objects $R=\left(L_{R}, \approx_{R}\right)$ where $\approx_{R}$ is reflexive, in other words the domain of $\approx_{R}$ coincides with the whole of $\left|L_{R}\right|$.

Similarly, SPEqu is the full subcategory of $\mathcal{P E} q u$ on those objects $K=\left(L_{K}, \approx_{K}\right)$ where $\approx_{K}$ is contained in the diagonal relation on $\left|L_{K}\right|$ —one may say that the relation $\approx_{K}$ is subreflexive.

Proposition 3.2 The restriction of the equivalence

$$
\mathcal{P E} q u \stackrel{Z}{\longrightarrow} \mathcal{E} q u
$$

to the subcategory $S P E q u$ determines an equivalence between $S P E q u$ and the image of the embedding $\mathcal{T}_{o p} \stackrel{Y}{\longrightarrow}$ Equ .

Proof It is enough to consider the diagram (1) and note that $\approx_{P}$ is subreflexive if and only if the map $\left(\mathrm{D}_{P}, \tau_{\text {sub }},=\right) \stackrel{\left[\mathrm{id}_{\mathrm{D}_{P}}\right]}{\longrightarrow} Z(P)$ is iso.

Remark 3.3 A condition similar to that used in the proof of Proposition 3.2 characterises a full subcategory $\mathcal{R E} q u$ of $\mathcal{E} q u$ equivalent to $R \mathcal{P E} q u$ : the objects of $\mathcal{R E} q u$ are those equilogical spaces $E$ for which there is an algebraic lattice $L$ and a regular epi $(L,=) \longrightarrow E$.

Notation 3.4 In line with the notation used in Remark 3.3 we shall write $\mathcal{S E q u}$ for the closure under isos of the image of the embedding $\mathcal{T}_{o p} \stackrel{Y}{Y}^{\longrightarrow}$ Equ.

Proposition 3.5 Let $K$ be an object in SPEqu and let $R$ be an object in RPEqu. Then:

(i) $R^{K}$ is in $R \mathcal{P E q u}$.

(ii) $K^{R}$ is in SPEqu.

Proof By Proposition 2.2(ii), for $f, f^{\prime}: L_{K} \longrightarrow L_{R}$, it is $f \approx_{R^{K}} f^{\prime}$ if and only if:

$$
\text { for all } a, a^{\prime} \in\left|L_{K}\right| \quad \text { if } a \approx_{K} a^{\prime} \text { then } f(a) \approx_{R} f^{\prime}\left(a^{\prime}\right)
$$

The two statements follow easily: For (i), when $\approx_{K}$ is subreflexive, $a \approx_{K} a^{\prime}$ is equivalent to $a \in \mathrm{D}_{K}$ and $a=a^{\prime}$, hence it is certainly $f \approx_{R^{K}} f$ for any $f: L_{K} \longrightarrow L_{R}$. For (ii), consider that by hypothesis $a \approx_{R} a$ for every $a \in\left|L_{R}\right|$. So, for every $f, f^{\prime}: L_{R} \longrightarrow L_{K}$ such that $f \approx_{K^{R}} f^{\prime}$, it follows that $f(a) \approx_{K} f^{\prime}(a)$ for every $a \in\left|L_{R}\right|$. Since $\approx_{K}$ is subreflexive, one has that $f=f^{\prime}$. 
Corollary 3.6 Let $P$ be an object in $\mathcal{P E} q u$. Then:

(i) If $\approx_{P}$ is subreflexive, then $\approx_{S^{P}}$ is reflexive.

(ii) If $\approx_{P}$ is reflexive, then $\approx_{S^{P}}$ is subreflexive.

Corollary 3.7 The functor $\mathrm{S}^{(-)}: \mathcal{E} q u \rightarrow \mathcal{E} q u^{\text {op }}$ applies the subcategory SEqu into $\mathcal{R E} q \mathfrak{u}^{o p}$ and viceversa, the subcategory $R \mathcal{E} q u$ into $S \mathcal{E} q u^{o p}$.

\section{The algebraic theory of an object}

We have seen in the previous section that the description of the monad $\mathbf{O}^{\mathbf{2}}$ can be performed in the internal language of the Cartesian closed category $\mathcal{C}$. In fact, those functor and natural transformations can be internalised in the sense of enriched categories, see Kock [14], Linton [16, 17], Power [21] and Street [25].

We refer the reader to previous references and to Kelly [13] for the notions of enriched category theory, in particular of monads in the enriched situation, or as they are called strong monads. Recall that a Cartesian closed category $\mathcal{C}$ has a canonical $\mathcal{C}$-enrichment given by the exponentials:

$$
\begin{gathered}
C^{\mathrm{op}} \times C \longrightarrow C^{\longrightarrow} \\
\langle C, D\rangle \longmapsto D^{C}
\end{gathered}
$$

In fact, this can be done for any symmetric monoidal closed category, but that kind of generality is not needed for the purposes of the present study. In the $\lambda$-notation, a composition arrow

$$
C^{B} \times D^{C} \stackrel{c_{B, C, D}}{\longrightarrow} D^{B}
$$

is given by the term

$$
\lambda x: B . g \cdot(f \cdot x) \quad \text { in context } f: C^{B}, g: D^{C}
$$

and, for $T$ the terminal object in $\mathcal{C}$, identities are $i_{A}: T \rightarrow C^{C}$ given by the term $\lambda x: C . x$ in the empty context. From now on, we may drop the application dot in case doing so generates no confusion, eg write $g(f x)$ in the term above.

We shall adopt a standard notation for the enriched homsets, and for objects $A$ and $A^{\prime}$ in the enriched $\mathcal{C}$-category $\mathcal{A}$ we write $\mathcal{A}\left[A, A^{\prime}\right]$ for the $\mathcal{C}$-object of $\mathcal{A}$-arrows from $A$ to $A^{\prime}$. So the $\mathcal{C}$-enrichment of the Cartesian closed category $\mathcal{C}$ is $C[C, D]=D^{C}$, and for 
arrows $f: C^{\prime} \rightarrow C$ and $g: D \rightarrow D^{\prime}$ in $\mathcal{C}$ the $\mathcal{C}$-arrow $C[f, g]: C[C, D] \rightarrow C\left[C^{\prime}, D^{\prime}\right]$ is given by the $\lambda$-term

$$
C[f, g](h)=\lambda x: C^{\prime} \cdot g[(h \cdot f) / y] \quad \text { in context } h: D^{C}=C[C, D]
$$

where $f$ and $g$ are in context $x: C^{\prime}$ and $y: D$ respectively. With respect to the canonical enrichment, $\mathcal{C}$ has $\mathcal{C}$-tensors and $\mathcal{C}$-cotensors given by product and power, respectively, since:

$$
C[I, C[C, D]] \stackrel{\sim}{\longrightarrow} C[I \times C, D] \stackrel{\sim}{\sim} C[C \times I, D] \stackrel{\sim}{\longrightarrow} C[C, C[I, D]]
$$

Note that every object of $\mathcal{C}$ is a tensor of the terminal object $T$. Similarly, the $\mathcal{C}$-enrichment of the category $\mathcal{C}^{\mathrm{op}}$ is $\mathcal{C}^{\mathrm{op}}[C, D]=C^{D}$.

Recall also that, for a fixed object $O$ in $\mathcal{C}$, the monad $\mathbf{O}^{2}$ is $\mathcal{C}$-enriched: the action of the functor $O^{2}$ on arrows, for every pair of objects $C$ and $D$ in $C$, is the $\mathcal{C}$-arrow

$$
C[C, D] \stackrel{O_{X, Y}^{2}}{\longrightarrow} C\left[O^{2}(C), O^{2}(D)\right]
$$

given by the term

$$
\lambda U: O^{2}(C) .\left(\lambda a: O^{1}(D) . U(\lambda x: C \cdot a(f x))\right) \text { in context } f: C[C, D],
$$

and there are commutative diagrams involving the natural transformations $\eta$ and $\mu$ as follows

$$
C[C, D] \stackrel{O_{C, D}^{2}}{\longrightarrow} C\left[O^{2}(C), O^{2}(D)\right]
$$

(2)

$$
\begin{gathered}
C[C, D] \stackrel{O_{C, D}^{2}}{\longrightarrow} C\left[O^{2}(C), O^{2}(D)\right] \\
O_{C, D}^{4} \downarrow \\
C\left[O^{4}(C), O^{4}(D)\right] \underset{C\left[C, \mu_{D}\right]}{\longrightarrow} C\left[O^{4}(C), O^{2}(D)\right]
\end{gathered}
$$

which internalise the standard (Set-enriched) commutative diagrams.

Remark 4.1 There is a very elegant analysis of this kind of monads in Kock [14] with an elementary characterisation of the conditions for enrichment given by the notion of strong monad.

Journal of Logic \& Analysis 11:FT5 (2019) 
We are interested in giving a presentation of the category of algebras for the monad $\mathbf{O}^{\mathbf{2}}$ in terms of certain enriched functors along the lines of Dubuc [6, 7] and Power [21]; see also Kelly [12]. The intuition about that presentation is that a $\mathcal{C}$-enriched monad is an abstract presentation of an algebraic theory (in a suitable internal sense) and it hinges on the parallel between the Kleisli category of a monad and the terms of a theory; see Streicher and Reus [26].

Remark 4.2 The precise sense in which to consider an "internal" algebraic theory requires the review of a few constructions on a $\mathcal{C}$-enriched monad which appear in the references. Unfortunately we have not been able to single out the explicit result we need, see Theorem 4.11. So, although the interest of the present paper is for monads of the form $\mathbf{O}^{2}$, in the following we sketch that result for an arbitrary $\mathcal{C}$-enriched monad $\mathbf{M}=(M, \eta, \mu)$ on $\mathcal{C}$.

First we briefly recall the notions of enriched Kleisli category and enriched EilenbergMoore category of an enriched monad. The Kleisli category $\mathcal{C}_{\mathbf{M}}$ of the monad $\mathbf{M}$ consists of the same objects as $\mathcal{C}$, but an arrow $t: X \rightarrow Y$ is an arrow $t: X \rightarrow M(Y)$ in $C$. The composition of $t: X \rightarrow Y$ and $s: Y \rightarrow Z$ is defined by the composition in $C$ of the following three arrows:

$$
X \stackrel{t}{\longrightarrow} M(Y) \stackrel{M(s)}{\longrightarrow} M^{2}(Z) \stackrel{\mu_{Z}}{\longrightarrow} M(Z)
$$

Taking advantage of the enriched monad, the Kleisli category has a $\mathcal{C}$-enrichment given by $\mathcal{C}_{\mathbf{M}}[X, Y]=\mathcal{C}[X, M(Y)]$. Also the $\mathcal{C}$-enriched Kleisli category inherits $\mathcal{C}$-tensors from $C$ since

$$
\mathcal{C}\left[I, \mathcal{C}_{\mathbf{M}}[X, Y]\right]=\mathcal{C}[I, \mathcal{C}[X, M(Y)]] \stackrel{\sim}{\longrightarrow} \mathcal{C}[I \times X, M(Y)]=\mathcal{C}_{\mathbf{M}}[I \times X, Y]
$$

quite similarly to how it inherits colimits from $\mathcal{C}$. Like in $\mathcal{C}$, every object of $\mathcal{C}_{\mathbf{M}}$ is a tensor of the terminal object $T$.

There is an identity-on-object, $\mathcal{C}$-enriched functor from $\mathcal{C}$ to $\mathcal{C}_{\mathbf{M}}$ which maps an arrow $f: X \rightarrow Y$ of $\mathcal{C}$ to the arrow $\eta_{Y} \circ f: X \rightarrow Y$ in $\mathcal{C}_{M}$.

Let $\mathcal{L}(\mathbf{M})$ be the opposite $\mathcal{C}$-enriched category $\mathcal{C}_{\mathbf{M}}$.

Remark 4.3 The category $\mathcal{L}(\mathbf{M})$ has $\mathcal{C}$-cotensors. Every object of $\mathcal{L}(\mathbf{M})$ is a cotensor of the object $T$ by the natural iso $V \simeq V \pitchfork T$.

Remark 4.4 Note that, in the case of a (standard) monad on the category Set for an algebraic theory, the Kleisli category is the category of free algebras and homomorphisms of the theory. 
For a monad of the form $\mathbf{O}^{2}$ there is an isomorphic presentation of $\mathcal{L}\left(\mathbf{O}^{2}\right)$ which will be useful for section 5. For $O$ an object in $\mathcal{C}$, let $\mathcal{T}(O)$ be the $C$-enriched category with the same object as $\mathcal{C}$-so the same objects as $\mathcal{C}_{\mathbf{O}^{2}}$ and $\mathcal{L}\left(\mathbf{O}^{2}\right)$-and let $\mathcal{T}(O)[V, W]=\mathcal{C}\left[O^{V}, O^{W}\right]$. Composition and identities are as in $\mathcal{C}$. Clearly, $\mathcal{T}(O)$ is equivalent to the full $C$-enriched subcategory of $C$ on the objects of the form $O^{V}$.

Proposition 4.5 Let $O$ be an object in $C$. Then there is an isomorphism of $C$-enriched categories $H: \mathcal{L}\left(\mathbf{O}^{2}\right) \longrightarrow \mathcal{T}(O)$.

Proof The functor $H$ is the identity on the objects of $\mathcal{L}\left(\mathbf{O}^{2}\right)$. To define the $C-$ enriched action on the arrows $H_{V, W}: \mathcal{L}\left(\mathbf{O}^{2}\right)[V, W] \rightarrow \mathcal{T}(O)[H(V), H(W)]$, consider the composite:

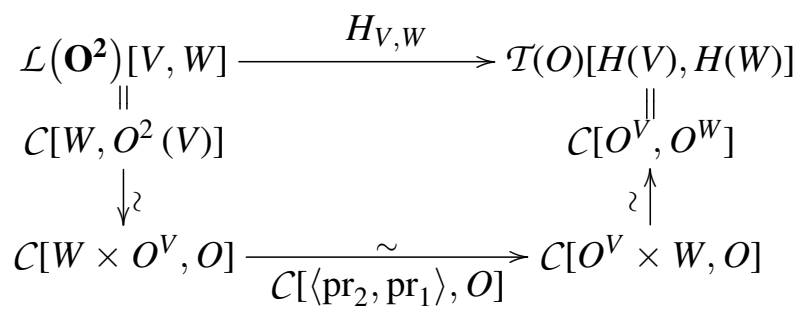

In $\lambda$-notation, $H_{V, W}$ is the term

$$
\lambda U: O^{V} \cdot \lambda y: W .(t \cdot y) \cdot U \quad \text { in context } t: \mathcal{L}\left(\mathbf{O}^{2}\right)[V, W]
$$

where one sees clearly that the "two arguments"of $t-y: W$ and $U: O^{V}$-get swapped. It is straightforward to check that the assignment $H$ is a $\mathcal{C}$-enriched functor. Moreover, since $H_{V, W}$ is iso, the functor is fully faithful. Hence $H$ is iso.

The Eilenberg-Moore category $\mathcal{C}^{\mathbf{M}}$ has objects the M-algebras, ie pairs $\mathcal{A}=$ $(A, \alpha: M(A) \rightarrow A)$ of an object $A$ and an arrow $\alpha: M(A) \rightarrow A$ in $C$ such that the diagrams
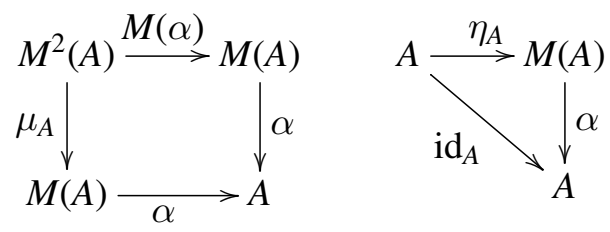

commute, and an arrow $h:(A, \alpha) \rightarrow(B, \beta)$ is an arrow $h: A \rightarrow B$ in $C$ which is a 
M-homomorphism, ie the following is a commutative diagram:

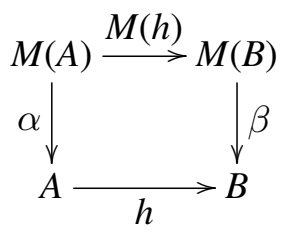

Example 4.6 For a fixed object $O$ in $\mathcal{C}$, an example of an $O^{2}$-algebra is $\mathcal{O}=\left(O, j_{O}\right)$ where $j_{O}: O^{2}(O) \rightarrow O$ denotes evaluation at $i_{O}$, ie the composite

$$
O^{2}(O) \stackrel{\left\langle\mathrm{id}_{O^{2}(O)}, i_{O} !\right\rangle}{\longrightarrow} O^{2}(O) \times O \stackrel{\mathrm{ev}}{\longrightarrow} O
$$

for !: $O^{2}(O) \rightarrow T$ the unique arrow to the terminal object, which can be written in $\lambda$-notation as $\phi \cdot(\lambda x: O . x)$ in context $\phi: O^{2}(O)$.

Remark 4.7 In case $C$ has equalisers, the Eilenberg-Moore category $C^{\mathbf{M}}$ has the $\mathcal{C}$-enrichment which, for $\mathbf{M}$-algebras $\mathcal{A}=(A, \alpha)$ and $\mathcal{B}=(B, \beta)$, is given by (a choice of) the equaliser

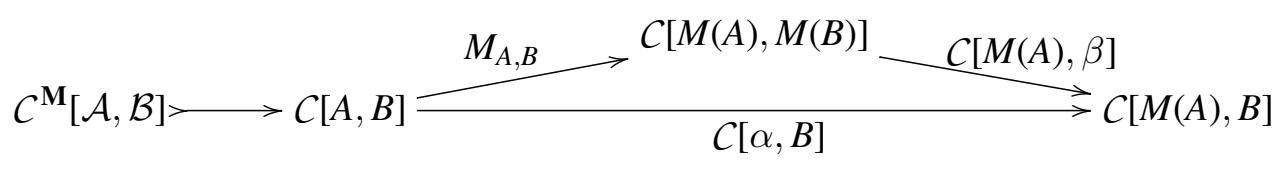

which internalises the commutativity condition for $\mathbf{M}$-homomorphisms; see Dubuc [7]. Such an enrichment inherits cotensors from $\mathcal{C}$. But in the general case of a category $\mathcal{C}$ with finite products that enrichment need not be available.

Remark 4.8 In the case of a (standard) monad on the category Set for an algebraic theory considered in Remark 4.4, the category $\mathcal{L}(\mathbf{M})$, being the opposite of the category of free algebras and homomorphisms between them, can be considered equivalently as the sets $V$ of the generators - think of variables. With that point of view, a $\mathcal{L}(\mathbf{M})$-arrow $t: V \rightarrow W$ is a $W$-list of terms of the theory written in the variables (ie the elements) of $V$ and the composition $V \stackrel{t}{\longrightarrow} W \stackrel{s}{\longrightarrow} U$ in $\mathcal{L}(\mathbf{M})$ is substitution of the variables $W$ in the terms of the $U$-list with the $W$-list of terms.

Notation 4.9 We recall when a $\mathcal{C}$-enriched functor $F: \mathcal{A} \longrightarrow \mathcal{B}$ preserves cotensors since we shall need it to prove Theorem 4.11. For objects $X$ in $\mathcal{A}$ and $I$ in $\mathcal{C}$, write $I \pitchfork X$ for the cotensor $X$ by $I$ and consider the universal $I$-family of $\mathcal{A}$-arrows 
$p_{I, X}: I \rightarrow \mathcal{A}[I \pitchfork X, X]$ of the cotensor $I \pitchfork X$, obtained by the exponential adjunction from the composite arrow

$$
\frac{\operatorname{id}_{I \pitchfork X}}{\longrightarrow} \mathcal{A}[I \pitchfork X, I \pitchfork X] \longrightarrow C[I, \mathcal{A}[I \pitchfork X, X]]
$$

from the terminal object $T$ of $\mathcal{C}$. Preservation of cotensors requires that $F$ transform each universal family $p_{I, X}$ into another such, in other words that the $\mathcal{B}$-arrow obtained by exponential adjunction from

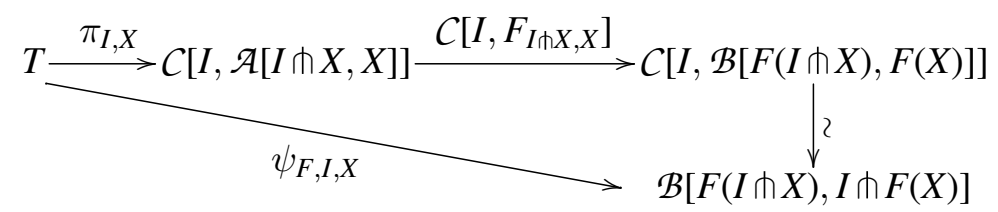

is iso, necessarily natural. In case $\mathcal{B}$ is $\mathcal{C}$, one can use $\lambda$-notation to write the adjunct $q_{F, I, X}: F(I \pitchfork X) \rightarrow I \pitchfork F(X)$ of $\psi_{F, I, X}$ as:

$$
\lambda i: I .\left(F\left(p_{I, X} \cdot i\right) \cdot a\right) \text { in context } a: F(I \pitchfork X)
$$

Following Dubuc [6] and Power [21] a model of $\mathcal{L}(\mathbf{M})$ is a $\mathcal{C}$-enriched functor $F: \mathcal{L}(\mathbf{M}) \longrightarrow \mathcal{C}$ which preserve cotensors.

Example 4.10 An $\mathbf{M}$-algebra $\mathcal{A}=(A, \alpha: M(A) \rightarrow A)$ determines a standard example of a $\mathcal{C}$-enriched, cotensor-preserving functor $\mathcal{A}^{(-)}: \mathcal{L}(\mathbf{M}) \longrightarrow \mathcal{C}$, see [6, 21]. It is defined as follows: on an object $V$ in $\mathcal{L}(\mathbf{M})$, the value $\mathcal{A}^{V}$ is $\mathcal{C}$ [V,A]. The $\mathcal{C}$-enriched action of $\mathcal{A}^{(-)}$

$$
\mathcal{L}(\mathbf{M})[V, W] \stackrel{\mathcal{A}^{(-)} V, W}{\longrightarrow} C[C[V, A], C[W, A]]
$$

is given by the $\lambda$-term

$$
\lambda f: \mathcal{C}[V, A] \cdot \mathcal{C}[t, \alpha] \cdot\left(M_{V, A} \cdot f\right) \text { in context } t: \mathcal{C}[V, W] .
$$

The proof that the assignment is indeed a functor is direct, though laborious, as it involves the categorical structure of $\mathcal{L}(\mathbf{M})=\left(\mathcal{C}_{\mathbf{M}}\right)^{\text {op }}$ and the conditions in (2). It is immediate to see that $\mathcal{A}^{(-)}$preserves cotensors as

$$
\mathcal{A}^{I \pitchfork V}=C[I \times V, A] \stackrel{\sim}{\longrightarrow} C[I, C[V, A]]=C\left[I, \mathcal{A}^{V}\right],
$$

and it is easy to check that, given an $\mathbf{M}$-homomorphism $h: \mathcal{A} \rightarrow \mathcal{B}$, post-composition with $h$

$$
\mathcal{A}^{V}=\mathcal{C}[V, A] \stackrel{\mathcal{C}[V, h]}{\longrightarrow} \mathcal{C}[V, B]=\mathcal{B}^{V}
$$

is a natural transformation. 
The result mentioned in Remark 4.2 is the statement that the examples in 4.10 are the most general.

Theorem 4.11 Let $\mathcal{C}$ be a Cartesian closed category and let $\mathbf{M}=(M, \eta, \mu)$ be $\mathcal{C}$-enriched monad on $\mathcal{C}$. Then the functor that assign to an $\mathbf{M}$-algebra $\mathcal{A}$ the $\mathcal{C}$-enriched, cotensor-preserving functor $\mathcal{A}^{(-)}: \mathcal{L}(\mathbf{M}) \longrightarrow \mathcal{C}$ is an equivalence of categories between $\mathcal{C}^{\mathbf{M}}$ and the full subcategory of the functor category $[\mathcal{L}(\mathbf{M}), \mathcal{C}]$ on the $\mathcal{C}$-enriched, cotensor-preserving functors.

Proof For the sake of space saving, in the proof write the category $\mathcal{L}(\mathbf{M})$ simply as $\mathcal{T}$. Fix a $\mathcal{C}$-enriched, cotensor-preserving functor $F: \mathcal{T} \longrightarrow \mathcal{C}$. Hence, for every object $I$ in $\mathcal{C}$ and every object $V$ in $\mathcal{T}$, the $\mathcal{C}$-arrow $\psi_{F, I, V}: F(I \pitchfork V) \longrightarrow C[I, F(V)]$ is natural iso. Since every object in $\mathcal{T}$ is a cotensor of the terminal object $T$, the value $F(T)$ determines the functor $F$ up to a natural isomorphism. By Remark 4.3 there is a natural iso $V \simeq V \pitchfork T$. So we can identify $V$ and $V \pitchfork T$ in $\mathcal{T}$, and $F(V)$ and $C[V, F(T)]$ in $C$. Also, to simplify notation, write $F(T)$ as $A$, so that:

$$
F_{V, W}: \mathcal{T}[V \pitchfork T, W \pitchfork T] \rightarrow \mathcal{C}[C[V, A], C[W, A]]
$$

Given any $\mathcal{T}$-arrow $t: V \rightarrow W$, there is a commutative diagram

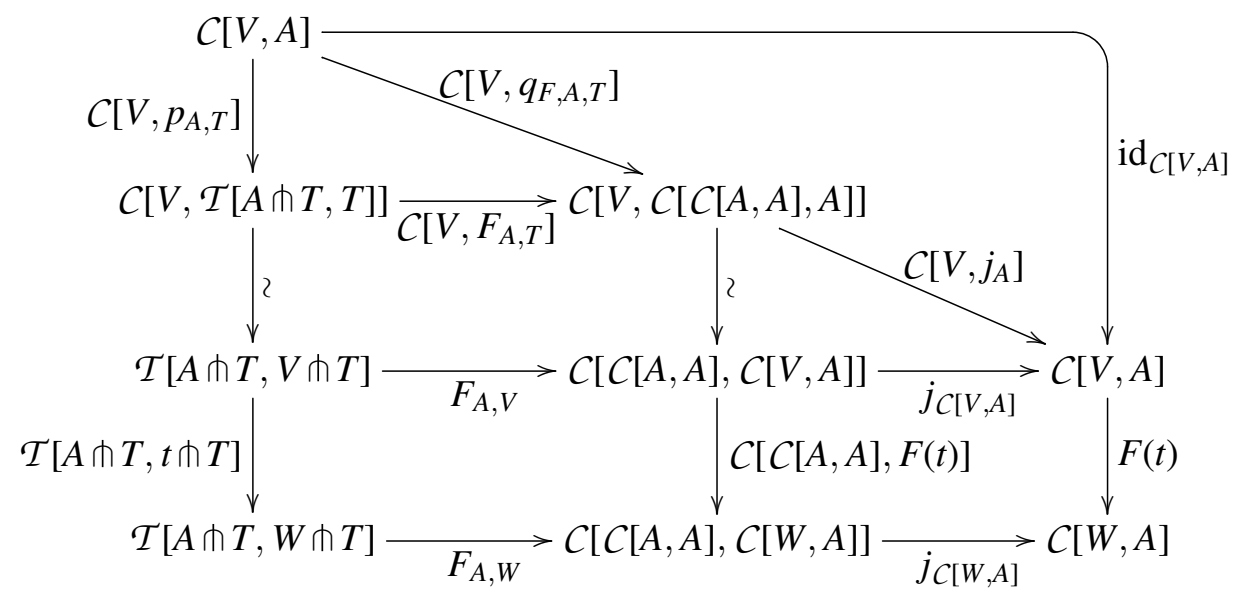

where, like in Example 4.6, the arrow $j_{C}: C[C[A, A], C] \rightarrow C$ denotes evaluation at the identity $i_{A}$, ie the composite

$$
C[C[A, A], C] \stackrel{\left\langle\operatorname{id}_{C[C[A, A], C]}, i_{A} !\right\rangle}{\longrightarrow} C[C[A, A], C] \times C[A, A] \stackrel{\mathrm{ev}}{\longrightarrow} C
$$

Remark 4.12 As already mentioned, there are results in the literature related to Theorem 4.11, for instance Theorem III in [6] and Theorem 3.4 in [21]. The reader may find more details about this in Santamaria's masters thesis [23]. 
In the particular case of the monad of an object $O$ in $\mathcal{C}$, one can see the models $F: \mathcal{T}(O) \rightarrow \mathcal{C}$ as an interpretation of all the operations $O^{V} \rightarrow O$ on $O$ available in $C$ which satisfies all identities that the operations satisfy on $O$. Or, turning things around, one can see $\mathcal{T}(O)$ as the algebraic theory of all the operations on $O$. And Theorem 4.11 states that the models are precisely the $\mathbf{O}^{2}$-algebras.

In case $C$ has equalisers, hence finite limits, by Remark 4.7 the Eilenberg-Moore category $C^{\mathbf{O}^{2}}$ is $\mathcal{C}$-enriched with cotensors. In particular, the natural isomorphism of cotensors

$$
C\left[I, C^{\mathbf{O}^{2}}[\mathcal{A}, \mathcal{O}]\right] \stackrel{\sim}{\longrightarrow} C^{\mathbf{O}^{2}}\left[\mathcal{A}, \mathcal{O}^{I}\right]=\left(C^{\mathbf{O}^{2}}\right)^{\mathrm{op}}\left[\mathcal{O}^{I}, \mathcal{A}\right]
$$

for objects $I$ in $\mathcal{C}$ and $\mathcal{A}$ in $\mathcal{C}^{\mathbf{O}^{2}}$, gives a $\mathcal{C}$-enriched adjunction:

$$
\left(C^{\mathbf{O}^{2}}\right)^{\mathrm{op}} \underset{\frac{\mathcal{O}^{(-)}}{C^{\mathbf{O}^{2}[-, \mathcal{O}]}}}{\perp} C
$$

In our case of interest, when $\mathcal{C}$ is $\mathcal{E} q u$ and $O$ is $\mathrm{S}$, if $T$ is a $\mathrm{T}_{0}$-space and $\widehat{T}$ denotes its soberification, then $\widehat{T} \longrightarrow \mathcal{E} q \mathcal{S}^{2}\left[\mathrm{~S}^{T}, \mathrm{~S}\right]$; see Bucalo and Rosolini [5].

\section{Global sections of $\mathbf{S}^{2}$-algebras}

By viewing an $\mathbf{S}^{\mathbf{2}}$-algebra $\mathcal{A}=\left(A, \alpha: \mathrm{S}^{2}(A) \longrightarrow A\right)$ as an $\mathcal{E} q u$-enriched, cotensorpreserving functor

$$
\begin{gathered}
\mathcal{T}(\mathrm{S}) \stackrel{\mathcal{A}^{(-)}}{\longrightarrow} \mathcal{E} q u \\
D \longmapsto
\end{gathered}
$$

from $\mathcal{T}(\mathrm{S})$ to $\mathcal{E} q u$, it is possible to distinguish some of the operations induced on the object $A$ by the $\mathbf{S}^{\mathbf{2}}$-structure $\alpha: \mathrm{S}^{2}(A) \longrightarrow A$ and determine the identities these satisfy. This is a slight abuse of notation since it should be $\mathcal{A}^{(-)}: \mathcal{L}\left(\mathbf{S}^{2}\right) \longrightarrow \mathcal{E} q u$, but by Proposition 4.5 the category $\mathcal{T}(\mathrm{S})$ is isomorphic to $\mathcal{L}\left(\mathbf{S}^{2}\right)$.

There is a useful, functorial way to analyse part of the structure given by a model $\mathcal{A}^{(-)}: \mathcal{T}(\mathrm{S}) \longrightarrow \mathcal{E} q u$. Let $\mathcal{D}$ be a subcategory of $\mathcal{T}(\mathrm{S})$ and write $I: \mathcal{D} \longleftrightarrow \mathcal{T}(\mathrm{S})$ the inclusion functor. Then the restriction functor

$$
\mathcal{D} \stackrel{I}{\longrightarrow} \mathcal{T}(\mathrm{S}) \stackrel{\mathcal{A}^{(-)}}{\longrightarrow} \mathcal{E} q u
$$


is a (Set-enriched) model of $\mathcal{D}$ in $\mathcal{E} q u$. If a syntactic presentation of the category $\mathcal{D}$ is available by means of a logical theory, then the functor $\mathcal{A}^{(-)} \circ I$ is a model of $\mathcal{D}$ with underlying object $A$. As an instance of this procedure, we show that every $\mathbf{S}^{\mathbf{2}}$-algebra $\mathcal{A}$ induces a distributive lattice structure on $A$.

Let $\mathcal{T}_{\text {Fin }}(\mathrm{S})$ be the full subcategory of $\mathcal{T}(\mathrm{S})$ whose objects are the discrete (finite) numerals.

Proposition 5.1 The category $\mathcal{T}_{\text {Fin }}(\mathrm{S})$ is the smallest subcategory of $\mathcal{T}(\mathrm{S})$ which contains the object 1 , is closed under finite products of $\mathcal{T}(\mathrm{S})$, and contains the arrows:

$$
\top: 0 \longrightarrow 1 \quad \perp: 0 \longrightarrow 1 \quad \wedge: 2 \longrightarrow 1 \quad \vee: 2 \longrightarrow 1
$$

Proof Each object $n$ in $\mathcal{T}_{\text {Fin }}(\mathrm{S})$ is the product of $n$ copies of 1 as $n=\overbrace{1+\ldots+1}^{n \text { times }}$. An arrow $f: n \longrightarrow m$ in $\mathcal{T}_{\text {Fin }}(\mathrm{S})$ is a continuous function $f: \mathrm{S}^{n} \longrightarrow \mathrm{S}^{m}$ between finite powers of $\mathrm{S}$. As such it is monotone and the four arrows in the statement are enough to generate by composition and pairing all such monotone maps.

Note that $\mathcal{T}_{\text {Fin }}(\mathrm{S})$ has an enrichment on the category $\mathcal{F}$ inSet of finite sets and functions and it has $\mathcal{F}$ inSet-cotensors.

Corollary 5.2 The category $\mathcal{T}_{\text {Fin }}(\mathrm{S})$ is the Lawvere algebraic theory of distributive lattices.

Proof The identities satisfied by the four arrows in Proposition 5.1 are precisely those given by commutative diagrams in $\mathcal{T}(\mathrm{S})$, in other words are precisely the identities satisfied by those operations in their interpretation as meet and join in $\mathrm{S}$.

Proposition 5.3 Every $\mathbf{S}^{2}$-algebra $\mathcal{A}$ inherits a structure of a (bounded) distributive lattice in $\mathcal{E} q u$ as given by the maps $\mathcal{A}^{\top}: \mathcal{A}^{0} \longrightarrow \mathcal{A}^{1}, \mathcal{A}^{\perp}: \mathcal{A}^{0} \longrightarrow \mathcal{A}^{1}, \mathcal{A}^{\wedge}: \mathcal{A}^{2} \longrightarrow$ $\mathcal{A}^{1}$ and $\mathcal{A}^{\vee}: \mathcal{A}^{2} \longrightarrow \mathcal{A}^{1}$.

Proof By Proposition 5.1, $\mathcal{T}_{\text {Fin }}(\mathrm{S})$ has finite products, computed by cotensors; so a cotensor-preserving functor from $\mathcal{T}(\mathrm{S})$ to $\mathcal{E} q u$ preserves such limits. So $\mathcal{A}^{0}$ is terminal in $\mathcal{E} q u$ and $\mathcal{A}^{2}$ is a product $A \times A$ in $\mathcal{E} q u$. Functoriality ensures that the operations in 
the statement satisfy all the commutative diagrams in which they appear in $\mathcal{T}(\mathrm{S})$. For instance, distributivity of $\vee$ over $\wedge$ is the commutative diagram

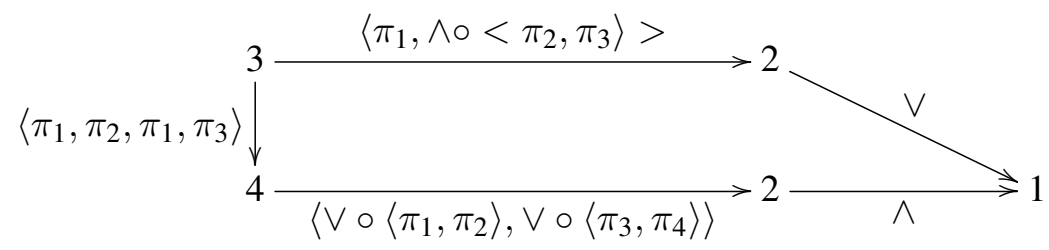

in $\mathcal{T}(\mathrm{S})$. Therefore, $\mathcal{A}^{(-)}$transforms it in the corresponding commutative diagram involving the operations on $A$.

In fact, in the following we strengthen Proposition 5.3 to show that every $\mathbf{S}^{\mathbf{2}}$-algebra has a unique structure of a frame. In order to do that, we introduce two other full subcategories of $\mathcal{T}(\mathrm{S})$ : the full subcategory $\mathcal{T}_{\text {FinPos }}(\mathrm{S})$ of $\mathcal{T}(\mathrm{S})$ on the finite posets (each considered with its Scott topology) and the full subcategory $\mathcal{T}_{\text {Set }}(\mathrm{S})$ on the discrete $\mathrm{T}_{0}$-spaces.

Proposition 5.4 The full subcategory $\mathcal{T}_{\text {FinPos }}(\mathrm{S})$ of $\mathcal{T}(\mathrm{S})$ is the smallest subcategory of $\mathcal{T}(\mathrm{S})$ which contains the object 1 , is closed under finite products and retracts and contains the arrows:

$$
\top: 0 \longrightarrow 1 \quad \perp: 0 \longrightarrow 1 \quad \wedge: 2 \longrightarrow 1 \quad \vee: 2 \longrightarrow 1
$$

Proof Because of Proposition 5.1, it is enough to show that any finite poset $P$ with the Scott topology is in $\mathcal{T}_{\text {FinPos }}(\mathrm{S})$. Let $n$ be the cardinality of $P$ and let $\ell: n \rightarrow P$ be a bijection. Consider the idempotent:

$$
\begin{aligned}
h: \mathrm{S}^{n} & \longrightarrow \mathrm{S}^{n} \\
U & \longmapsto\left[i \mapsto \bigvee_{\ell(n) \leq_{P} \ell(i)} U(n)\right]
\end{aligned}
$$

It is immediate to see that $\mathrm{S}^{P}$ is (isomorphic to) the distributive lattice of fixpoints of $h$.

The following result is reminiscent of the study in Hyland [11]; see also Fiore and Rosolini [8].

Corollary 5.5 The lattice structure on an $\mathbf{S}^{2}$-algebra $\mathcal{A}=\left(A, \alpha: \mathrm{S}^{2}(A) \longrightarrow A\right)$ depends only on the underlying object $A$. 
Proof The object $S$ is in $\mathcal{T}_{\text {FinPos }}(S)$; for instance, in $\mathcal{T}(\mathrm{S})$ it splits the idempotent on 2:

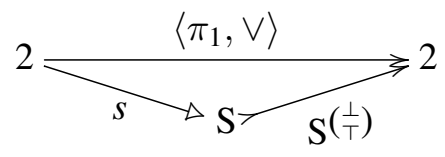

So the order on $A$ induced by the $\mathbf{S}^{\mathbf{2}}$-algebra is determined by the monic $A^{\left(\frac{\perp}{T}\right)}$

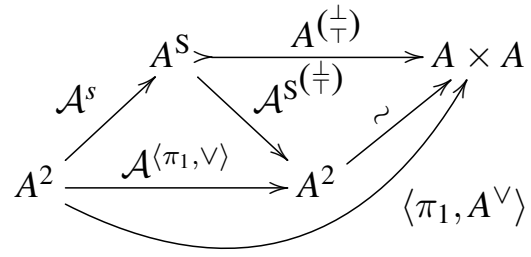

since the functor $\mathcal{A}^{(-)}$preserves the splitting in (3). But a lattice structure is uniquely determined by the order on the underlying $A$.

Proposition 5.6 The category $\mathcal{T}_{\text {Set }}(\mathrm{S})$ is the smallest subcategory of $\mathcal{T}(\mathrm{S})$ which contains the object 1 , is closed under arbitrary products and contains the arrows

$$
\top: 0 \longrightarrow 1 \quad \perp: 0 \longrightarrow 1 \quad \wedge: 2 \longrightarrow 1 \quad \vee: 2 \longrightarrow 1 \quad \bigvee_{I}: I \rightarrow 1
$$

where $I$ varies among arbitrary sets.

Proof Given a discrete $\mathrm{T}_{0}$-space $I$ and a subset $E \subseteq I$, let $i_{E}: E \hookrightarrow I$ be the inclusion map of the discrete topological space $E$ and let $j_{E}: S^{E} \longrightarrow S^{I}$ be the inclusion of the powerset of $E$ into the powerset of $I$. In $\mathcal{E} q u$, the diagram

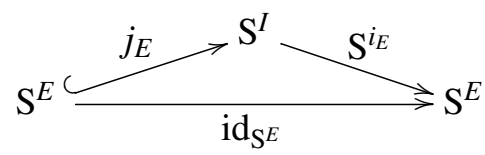

commutes producing every subset (equivalently, subspace) $E$ of $I$ as an idempotent of $I$ in $\mathcal{T}_{\mathrm{Set}}(\mathrm{S})$ :

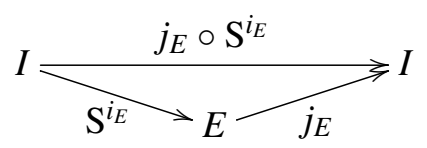

The two maps $S^{i_{E}}: I \longrightarrow E$ and $j_{E}: E \longrightarrow I$ are into products of 1 in $\mathcal{T}_{\text {Set }}(\mathrm{S})$; by evaluating at any element $x$ one sees that each component is either a projection or the constant map $\perp$ !: $E \longrightarrow 1$. Moreover the family

$$
\left(j_{E} \circ S^{i_{E}}: I \longrightarrow I\right)_{E \subseteq I, E \text { finite }}
$$


is directed and its join is the identity map on $I$ in $\mathcal{T}_{\text {Set }}(\mathrm{S})$.

Let now $f: I \longrightarrow 1$ be an arbitrary map in $\mathcal{T}_{\text {Set }}(\mathrm{S})$; in other words, a continuous function $f: \mathrm{S}^{I} \longrightarrow \mathrm{S}^{1}$ from a power of $\mathrm{S}$ into $\mathrm{S}$. Consider the diagram

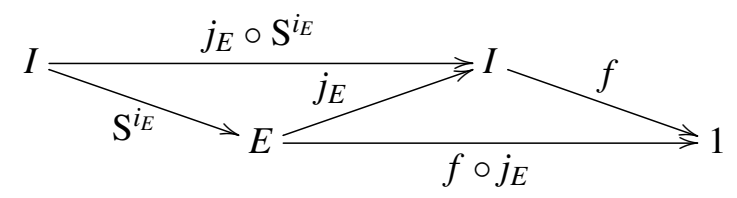

which displays the map $f$ is the directed join of the family of maps $\left(f \circ j_{E} \circ S^{i_{E}}\right): I \rightarrow 1$ as $E$ varies among the finite subsets of $I$. By Proposition 5.1, each map $f \circ j_{E}: E \longrightarrow 1$ can be obtained from the lattice structure and each map $S^{i_{E}}$ is obtained as a pairing of projections.

Corollary 5.7 Every $\mathbf{S}^{\mathbf{2}}$-algebra is an internal frame in $\mathcal{E} q u$.

Proof It is enough to note that the map which takes joins $\bigvee_{I}: S^{I} \longrightarrow S$ gives rise to a map $\bigvee_{I}: I \longrightarrow 1$ in $\mathcal{T}_{\text {Set }}(\mathrm{S})$ and that arbitrary joins are characterised by identities.

Corollary 5.8 The global section functor of equilogical spaces $\Gamma: \mathcal{E} q u \longrightarrow$ Set extends to a faithful functor $\mathcal{E} q u^{\mathbf{S}^{2}} \rightarrow \mathcal{F} r m$.

Proof Note that the discrete $\mathrm{T}_{0}$-spaces are the values of the left adjoint $\Delta: \operatorname{Set} \longrightarrow$ $\mathcal{E} q u$ of $\Gamma: \mathcal{E} q u \longrightarrow S e t$, in fact for every discrete $\mathrm{T}_{0}$-space $I$ there is an iso $I \stackrel{\sim}{\longrightarrow} \Delta(\Gamma(I))$ and that $\Gamma(\mathcal{E} q u[D, E])$ is exactly the (standard) homset of maps from $D$ to $E$.

\section{Final remarks and further directions}

The result in Corollary 5.8 may induce to consider that the category of $\mathbf{S}^{\mathbf{2}}$-algebras and homomorphisms resembles that of frames and frame-homomorphisms. But the analysis performed in the $\mathcal{E} q u$-enriched case seems to suggest a different situation: while an $\mathbf{S}^{\mathbf{2}}$-algebra bears a structure of a frame, there may be more to the structure than just that. And there may be more structure, or the structure that is induced by the order satisfies more properties than simply being a frame. Frosoni's Ph.D. thesis [9] contains further results about that.

A very interesting remark that derives from Proposition 5.6 is that the $\mathcal{E} q u$-enrichment reduces the infinitary algebraic structure of frames to a finitary situation where the only 
reference to the infinite is the existence of arbitrary products in the category $\mathcal{T}_{\text {Set }}(\mathrm{S})$. This suggests that it is possible to see the notion of frame as a finitary one from some appropriate, non-classical point of view. There is a similar approach in Hyland [11].

\section{Acknowledgements}

We would like to thank the editors of the special issue, Thierry Coquand, Maria Emilia Maietti and Erik Palmgren for their strong commitment, and an anonymous referee for very useful suggestions which helped improving the presentation.

GNSAGA and Correctness by Construction (EU 7th framework programme, grant no. PIRSES-GA-2013-612638) provided support for the research presented in the paper.

\section{References}

[1] A Bauer, L Birkedal, D S Scott, Equilogical spaces, Theoret. Comput. Sci. 315 (2004) 35-59; https://doi.org/10.1016/j.tcs.2003.11.012

[2] L Birkedal, A Carboni, G Rosolini, D Scott, Type theory via exact categories, from: "Proc. 13th Symposium in Logic in Computer Science", (V Pratt, editor), I.E.E.E. Computer Society, Indianapolis (1998) 188-198; https://doi.org/10.1109/LICS.1998.705655

[3] A Bucalo, G Rosolini, Repleteness and the associated sheaf, J. Pure Appl. Algebra 127 (1998) 147-151; https://doi.org/10.1016/S0022-4049(96)00151-X

[4] A Bucalo, G Rosolini, Completions, comonoids, and topological spaces, Ann. Pure Appl. Logic 137 (2006) 104-125; https://doi.org/10.1016/j.apal.2005.05.029

[5] A Bucalo, G Rosolini, Sobriety for equilogical spaces, Theoret. Comput. Sci. 546 (2014) 93-98; https://doi.org/10.1016/j.tcs.2014.03.002

[6] E J Dubuc, Enriched semantics-structure (meta) adjointness, Rev. Un. Mat. Argentina 25 (1970) 5-26

[7] E J Dubuc, Kan Extensions in Enriched Category Theory, volume 140 of Lecture Notes in Math., Springer-Verlag (1970); https://doi.org/10.1007/BFb0060485

[8] M Fiore, G Rosolini, Two models of Synthetic Domain Theory, J. Pure Appl. Algebra 116 (1997) 151-162; https://doi.org/10.1016/S0022-4049(96)00164-8

[9] G Frosoni, Equilogical Spaces, Frames and a Double-Power Monad, PhD thesis, Università degli studi di Genova (2017) 
G Frosoni, G Rosolini and A Santamaria

[10] G Frosoni, G Rosolini, Equilogical spaces and algebras for a double-power monad, Tblisi Mathematical Journal 10 (2017) 121-139; https://doi.org/10.1515/tmj-2017-0105

[11] J M E Hyland, First steps in Synthetic Domain Theory, from: "Category Theory '90', (A Carboni, M C Pedicchio, G Rosolini, editors), Lecture Notes in Math. 1488, Springer-Verlag, Como (1992) 131-156; https://doi.org/10.1007/BFb0084217

[12] G M Kelly, Review of [6], Math. Reviews (1975) MR0352213 (50 \#4700); http://www.ams.org/mathscinet-getitem?mr=0352213

[13] GM Kelly, Basic concepts of enriched category theory, volume 64 of London Math. Soc. Lecture Note Ser., Cambridge University Press (1982); http://www.tac.mta.ca/tac/reprints/articles/10/tr10.pdf

[14] A Kock, Strong functors and monoidal monads, Arch. Math. 23 (1972) 113-120; https://doi.org/10.1007/BF01304852

[15] J Lambek, P J Scott, Introduction to Higher Order Categorical Logic, Cambridge University Press (1986)

[16] F E J Linton, Relative functorial semantics: Adjointness results, from: "Category Theory, Homology Theory and their Applications, III (Battelle Institute Conference, Seattle, 1968, Vol. Three)", Springer, Berlin (1969) 384-418; https://doi.org/10.1007/BFb0081968

[17] F E J Linton, Review of [25], Math. Reviews (1973) MR0299653 (45 \#8701); http://www.ams.org/mathscinet-getitem?mr=0299653

[18] M Maietti, G Rosolini, Elementary quotient completion, Theory Appl. Categ. 27 (2013) 445-463; https://doi.org/10.1109/LICS.1998.705655

[19] M Maietti, G Rosolini, Quotient completion for the foundation of constructive mathematics, Log. Univers. 7 (2013) 371-402; https://doi.org/10.1007/s11787-013-0080-2

[20] M Maietti, G Rosolini, Unifying exact completions, Appl. Categ. Structures 23 (2015) 43-52; https://doi.org/10.1007/s10485-013-9360-5

[21] J Power, Enriched Lawvere theories, The Lambek Festschrift, Theory Appl. Categ. 6 (1999) 83-93; http://www.tac.mta.ca/tac/volumes/6/n7/n7.pdf

[22] G Rosolini, T Streicher, Comparing models of higher type computation, Electron. Notes Theor. Comput. Sci. 23 (1999) 159-165; https://doi.org/10.1016/S1571-0661(04) 00109-4

[23] A Santamaria, Frames and Equilogical Spaces, Master's thesis, Università degli studi di Genova (2015)

[24] D S Scott, A new category? Domains, spaces and equivalence relations, Unpublished Manuscript (1996)

[25] R Street, The formal theory of monads, J. Pure Appl. Algebra 2 (1972) 149-168; https://doi.org/10.1016/0022-4049(72)90019-9 
[26] T Streicher, B Reus, Classical logic, continuation semantics and abstract machines, J. Funct. Programming 8 (1998) 543-572; https://doi.org/10.1017/S0956796898003141

[27] P Taylor, Geometric and higher order logic in terms of abstract Stone duality, Theory Appl. Categ. 7 (2000) 284-338; http://www.tac.mta.ca/tac/volumes/7/n15/n15.pdf

[28] P Taylor, Sober spaces and continuations, Theory Appl. Categ. 10 (2002) No. 12, 248-300; http://www.tac.mta.ca/tac/volumes/10/12/10-12.pdf

[29] P Taylor, Subspaces in abstract Stone duality, Theory Appl. Categ. 10 (2002) No. 13, 301-368; http://www.tac.mta.ca/tac/volumes/10/13/10-13.pdf

[30] P Taylor, Computably based locally compact spaces, Log. Methods Comput. Sci. 2 (2006) 1:1, 70pp.; https://doi.org/10.2168/LMCS-2(1:1)2006

[31] P Taylor, A lambda calculus for real analysis, J. Log. Anal. 2 (2010) Paper 5, 115pp;; https://doi.org/10.4115/jla.2010.2.5

[32] P Taylor, Foundations for computable topology, from: "Foundational theories of classical and constructive mathematics", West. Ont. Ser. Philos. Sci. 76, Springer, Dordrecht (2011) 265-310; https://doi.org/10.1007/978-94-007-0431-2_14

[33] H Thielecke, Continuation semantics and self-adjointness, Electron. Notes Theor. Comput. Sci. 6 (1997); https://doi.org/10.1016/S1571-0661(05)80149-5

[34] S J Vickers, C F Townsend, A universal characterization of the double powerlocale, Theoret. Comput. Sci. 316 (2004) 297-321; https://doi.org/10.1016/j.tcs.2004.01.034

DIMA, via Dodecaneso 35, 16146 Genova, Italy

DIMA, via Dodecaneso 35, 16146 Genova, Italy

School of EECS, Queen Mary University of London, Mile End Road, London E1 4NS, UK

frosoni@dima.unige.it, rosolini@unige.it, a.santamaria@qmul.ac.uk

Received: 30 March $2017 \quad$ Revised: 30 Oct 2019 